\title{
Above-threshold ionization in the $x$-ray regime
}

\author{
Hari R. Varma, ${ }^{1}$ Marcelo F. Ciappina, ${ }^{2}$ Nina Rohringer, ${ }^{3}$ and Robin Santra ${ }^{1,4}$ \\ ${ }^{1}$ Argonne National Laboratory, Argonne, Illinois 60439, USA \\ ${ }^{2}$ Institute of High Performance Computing, 1 Fusionopolis Way \#16-16 Connexis, Singapore 138682, Singapore \\ ${ }^{3}$ Lawrence Livermore National Laboratory, Livermore, California 94551, USA \\ ${ }^{4}$ Department of Physics, University of Chicago, Chicago, Illinois 60637, USA
}

(Received 1 June 2009; published 30 November 2009)

\begin{abstract}
Two-photon above-threshold ionization processes in the x-ray regime are studied using atomic hydrogen as a model system. Within the minimal-coupling formalism of nonrelativistic quantum electrodynamics, two distinct interactions- $\hat{\mathbf{A}} \cdot \hat{\mathbf{p}}$ in second order and $\hat{\mathbf{A}}^{2}$ in first order-contribute to the two-photon absorption amplitude. The relative importance of these two interactions is assessed. It is found that above a photon energy of $6.8 \mathrm{keV}$, the contribution from $\hat{\mathbf{A}}^{2}$ to the total two-photon absorption cross section dominates. In this high-energy regime, above-threshold ionization is a nonsequential purely nondipole process. Rate equations are employed to calculate the probabilities of ionization by Compton scattering, one-photon absorption, and twophoton absorption.
\end{abstract}

DOI: 10.1103/PhysRevA.80.053424

PACS number(s): 32.80.Rm, 31.15.A-, 32.30.Rj, 41.60.Cr

\section{INTRODUCTION}

The advent of $x$-ray free-electron lasers [1-4] will open up hitherto unexplored frontiers in physics, chemistry, biology, and interdisciplinary areas such as materials science [5-10]. These new light sources will provide highly intense radiation with photon energies in the soft and hard $\mathrm{x}$-ray regimes. The Linac Coherent Light Source (LCLS) [2] at SLAC National Accelerator Laboratory has recently become available for user experiments and supplies $\sim 10^{12}$ photons in a sub-100-fs pulse in the $\mathrm{x}$-ray energy range between $800 \mathrm{eV}$ and $8 \mathrm{keV}$. This powerful tool makes it possible to study $\mathrm{x}$-ray multiphoton absorption processes.

A number of theoretical studies have been reported on the response of multielectron systems to such intense $\mathrm{x}$ rays [7-13]. Since the energy of an x-ray photon is typically high enough to ionize a bound electron via one-photon absorption, these studies focused on sequential multiphoton absorption processes, in which each absorbed photon ionizes a different bound electron in the system. X-ray interactions with electrons in the continuum-so-called free-free transitionswere neglected. In the high-intensity infrared, visible, and ultraviolet regimes, it has been demonstrated that, as a consequence of free-free transitions, photoelectrons may possess a kinetic energy that is higher than the photon energy minus the electron binding energy [14-20]. This effect is referred to as above-threshold ionization (ATI).

The purpose of this paper is to investigate the nature of ATI in the $\mathrm{x}$-ray regime. The high-intensity $\mathrm{x}$-ray studies mentioned earlier were based on the electric dipole approximation. In the x-ray regime, the wavelength of the radiation is comparable to the size of the atom. Thus, one may anticipate that nondipole effects play a significant role in X-ray ATI [21]. Within the minimal-coupling formalism of nonrelativistic quantum electrodynamics [22], electrons and photons are coupled via two distinct operators $\hat{\mathbf{A}} \cdot \hat{\mathbf{p}}$ and $\hat{\mathbf{A}}^{2}$, where $\hat{\mathbf{A}}$ is the vector potential in the Coulomb gauge, and $\hat{\mathbf{p}}$ is the canonical momentum of the electron. In the electric dipole approximation, which within the minimal-coupling formal- ism corresponds to neglecting the photon momentum [22], $\hat{\mathbf{A}}^{2}$ is independent of the electron degrees of freedom and can, therefore, not induce transitions between electronic eigenstates. Particularly, ATI at long wavelengths may be fully understood in terms of the $\hat{\mathbf{A}} \cdot \hat{\mathbf{p}}$ interaction. In the following, we demonstrate that at high photon energies, the nature of ATI is fundamentally different. Above a photon energy of $6.8 \mathrm{keV}$, two-photon ATI of atomic hydrogen is dominated by $\hat{\mathbf{A}}^{2}$. Atomic units are used throughout, unless otherwise noted.

\section{FORMALISM}

Atomic hydrogen is an ideal system for investigating $\mathrm{x}$-ray ATI because there is only one electron and, therefore, it is guaranteed that in a two-photon process, the electron will absorb both photons. Two-photon processes in hydrogen and hydrogenlike systems have been studied theoretically in Refs. [23-26] and experimentally in Refs. [27-29]. However, to the best of our knowledge, no work on ATI in the highintensity $\mathrm{x}$-ray regime has been reported.

We employ the time-dependent perturbation theory [22] to analyze the x-ray two-photon ATI process. The unperturbed Hamiltonian $\hat{H}_{0}$ is the sum of the atomic Hamiltonian and the electromagnetic-field Hamiltonian. The matter-field interaction, which in Coulomb gauge reads as

$$
\hat{H}_{I}=\alpha \hat{\mathbf{A}} \cdot \hat{\mathbf{p}}+\frac{\alpha^{2}}{2} \hat{\mathbf{A}}^{2},
$$

is treated as a perturbation. In Eq. (1), $\alpha$ is the fine-structure constant. Before the interaction with the $\mathrm{x}$-ray photons, the hydrogen atom is assumed to be in the atomic eigenstate $\left|\Psi_{i}\right\rangle$ with eigenenergy $\varepsilon_{i}$. We assume that the initial state of the photon field may be represented by a single-mode Fock state $|N\rangle$. Here, $N$ indicates the number of photons in the single mode. In the interaction picture, the initial state of the atomphoton system can be written as the direct product 


$$
\left|\Psi_{i}^{N}\right\rangle=\left|\Psi_{i}\right\rangle|N\rangle .
$$

This is an eigenstate of $\hat{H}_{0}$ with eigenvalue $\varepsilon_{i}+N \omega$, where $\omega$ is the photon energy.

Assuming the initial condition

$$
\lim _{t \rightarrow-\infty}|\Psi, t\rangle=\left|\Psi_{i}^{N}\right\rangle,
$$

the time evolution of the state of the interacting atom-photon system in the interaction picture is

$$
\begin{aligned}
|\Psi, t\rangle= & {\left[1-i \int_{-\infty}^{t} d t_{1} \hat{H}_{I}^{\prime}\left(t_{1}\right)\right.} \\
& \left.-\int_{-\infty}^{t} d t_{1} \int_{-\infty}^{t_{1}} d t_{2} \hat{H}_{I}^{\prime}\left(t_{1}\right) \hat{H}_{I}^{\prime}\left(t_{2}\right)+\ldots\right]\left|\Psi_{i}^{N}\right\rangle,
\end{aligned}
$$

where

$$
\hat{H}_{I}^{\prime}(t)=e^{i \hat{H}_{0} t} \hat{H}_{I} e^{-\epsilon|t|} e^{-i \hat{H}_{0} t} .
$$

The infinitesimal $\epsilon>0$ is introduced to ensure that the perturbation disappears long before $(t \rightarrow-\infty)$ and long after $(t \rightarrow \infty)$ the interaction between the atom and the $\mathrm{x}$ rays. This particular procedure is referred to as adiabatic switching [30].

The two-photon transition amplitude

$$
S_{i f}=\lim _{t \rightarrow \infty}\left\langle\Psi_{f}^{N-2} \mid \Psi, t\right\rangle
$$

is obtained by projecting $|\Psi, t\rangle$ onto the final state

$$
\left|\Psi_{f}^{N-2}\right\rangle=\left|\Psi_{f}\right\rangle|N-2\rangle .
$$

Using standard methods (see, for instance, Ref. [31]), we obtain from the two-photon transition amplitude $S_{i f}$ the twophoton transition rate $\Gamma_{i f}$. Finally, the total x-ray two-photon ATI cross section $\sigma_{\text {total }}^{(2)}$ is calculated by summing over all final states. The final states are energy-normalized [32] continuum states with orbital angular momentum quantum number $l_{f}$ and projection quantum number $m_{f}$. Hence,

$$
\sigma_{\text {total }}^{(2)}=\sum_{l_{f}, m_{f}} \int_{0}^{\infty} \frac{\Gamma_{i f}}{J^{2}} d \epsilon_{f}
$$

where

$$
J=\frac{1}{\alpha} \frac{N}{V}
$$

is the photon flux, and $V$ is the normalization volume. When evaluating Eq. (8), it is exploited that for $N \gg 1$, $N(N-1) \approx N^{2}$.

\section{TWO-PHOTON ATI CROSS SECTION}

Two-photon absorption is a first-order process with respect to $\hat{\mathbf{A}}^{2}$ and a second-order process with respect to $\hat{\mathbf{A}} \cdot \hat{\mathbf{p}}$. Thus, from Eqs. (1)-(9), the total two-photon ATI cross section for linearly polarized photons with energy $\omega$ and momentum $\mathbf{k}$ is obtained as

$$
\begin{aligned}
\sigma_{\text {total }}^{(2)}= & \frac{8 \pi^{3} \alpha^{2}}{\omega^{2}} \sum_{l_{f}, m_{f}} \mid \sum_{a} \frac{\left\langle\Psi_{f}\left|\hat{p}_{z} e^{i \mathbf{k} \cdot \mathbf{r}}\right| \Psi_{a}\right\rangle\left\langle\Psi_{a}\left|\hat{p}_{z} e^{i \mathbf{k} \cdot \mathbf{r}}\right| \Psi_{i}\right\rangle}{\varepsilon_{a}-\varepsilon_{i}-\omega-i \epsilon} \\
& -\left.\frac{1}{2}\left\langle\Psi_{f}\left|e^{2 i \mathbf{k} \cdot \mathbf{r}}\right| \Psi_{i}\right\rangle\right|_{\varepsilon_{f}=\varepsilon_{i}+2 \omega} ^{2} .
\end{aligned}
$$

Here $\left|\Psi_{i}\right\rangle,\left|\Psi_{a}\right\rangle$, and $\left|\Psi_{f}\right\rangle$ are, respectively, the initial, intermediate, and final states of the atomic system; $\varepsilon_{i}, \varepsilon_{a}$, and $\varepsilon_{f}$ are the corresponding energies. The final-state energy $\varepsilon_{f}$ equals $\varepsilon_{i}+2 \omega$. The polarization axis is chosen as the $z$ axis. The sum over intermediate states includes continuum states.

First, we study the importance of nondipole corrections in the first term of Eq. (10). This analysis is difficult because of the presence of free-free transition matrix elements. These matrix elements are numerically unstable in the length and velocity gauges [33]. We therefore use the nondipole Kramers-Henneberger formalism, which has been successfully employed in recent years [34-36]. The second term in Eq. (10), which is due to the $\hat{\mathbf{A}}^{2}$ interaction, is unaltered in the nondipole Kramers-Henneberger formalism, thus, allowing one to identify the $\hat{\mathbf{A}} \cdot \hat{\mathbf{p}}$ contribution to Eq. (10). For atomic hydrogen initially in its ground state $(i=1 s)$, we find that the nondipole contribution to the second-order $\hat{\mathbf{A}} \cdot \hat{\mathbf{p}}$ term in Eq. (10) is less than a percent, even at a photon energy as high as $8 \mathrm{keV}$.

We therefore continue our analysis of the first term of Eq. (10) using the electric dipole approximation (i.e., $\mathbf{k}=\mathbf{0}$ ). Disregarding for the moment the $\hat{\mathbf{A}}^{2}$ interaction and employing the identity

$$
\frac{1}{x-i \epsilon}=\mathrm{P} \frac{1}{x}+i \pi \delta(x)
$$

where $\mathrm{P}$ indicates that the principal value must be taken, the two-photon ATI cross section for $i=1 s$ may be written as

$$
\begin{aligned}
\sigma_{\hat{\mathbf{A}} \cdot \hat{\mathbf{p}}}^{(2)}= & \frac{8 \pi^{3} \alpha^{2}}{\omega^{2}} \sum_{l_{f}} \mid \mathrm{P} \int d \varepsilon \frac{\left\langle\Psi_{f}\left|\frac{\cos \vartheta}{r^{2}}\right| \Psi_{\varepsilon p}\right\rangle\left\langle\Psi_{\varepsilon p}\left|\frac{\cos \vartheta}{r^{2}}\right| \Psi_{1 s}\right\rangle}{\left(\varepsilon_{f}-\varepsilon\right)\left(\varepsilon-\varepsilon_{1 s}\right)\left(\varepsilon-\varepsilon_{1 s}-\omega\right)} \\
& +\left.\frac{i \pi}{\omega^{2}}\left\langle\Psi_{f}\left|\frac{\cos \vartheta}{r^{2}}\right| \Psi_{\tilde{\epsilon} p}\right\rangle\left\langle\Psi_{\tilde{\epsilon} p}\left|\frac{\cos \vartheta}{r^{2}}\right| \Psi_{1 s}\right\rangle\right|_{\varepsilon_{f}=\varepsilon_{1 s}+2 \omega} ^{2} .
\end{aligned}
$$

Here, $\vartheta$ is the polar angle with respect to the polarization axis, and $\tilde{\epsilon}=\varepsilon_{1 s}+\omega$. The angular momentum projection quantum numbers for the states appearing in Eq. (12) are zero.

At long wavelengths, the two-photon absorption is mediated largely by bound intermediate states. In the x-ray regime, however, the contributions from bound intermediate states are negligible (as we have numerically verified). These contributions are therefore not shown in Eq. (12). We further find that, at high photon energy, the contribution from the $\mathrm{P}$ part is negligible compared to the second term in Eq. (12). This second term, which we call the $\delta$ part, describes twophoton transitions in which the overall energy is conserved even in the intermediate state. At a photon energy of $800 \mathrm{eV}$, 


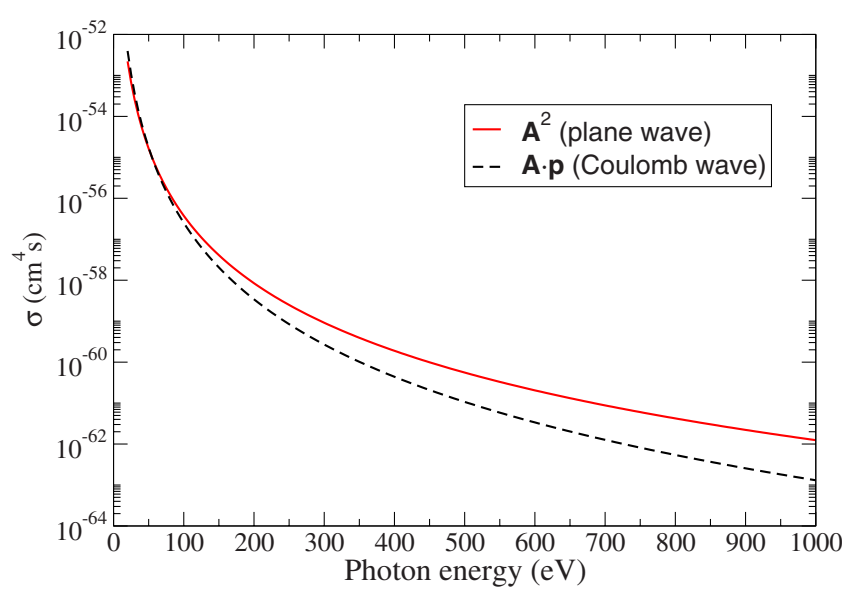

FIG. 1. (Color online) Contributions to the two-photon ATI cross section: $\hat{\mathbf{A}} \cdot \hat{\mathbf{p}}$ (dashed line); $\hat{\mathbf{A}}^{2}$ (solid line). The $\hat{\mathbf{A}}^{2}$ interaction is treated within the plane-wave model.

the contribution of the $\delta$ part is five orders of magnitude greater than the contribution of the $\mathrm{P}$ part. The $\mathrm{P}$ part is suppressed because of the slow variation in the dipole matrix elements with respect to energy. This makes the contributions to the integration from both sides of the pole at $\varepsilon=\varepsilon_{1 s}+\omega$ essentially equal in magnitude and, hence, cancel each other.

From these observations, we may conclude that the twophoton ATI cross section described by Eq. (12) scales approximately as $1 / \omega^{6}$. The numerically exact $\hat{\mathbf{A}} \cdot \hat{\mathbf{p}}$-induced two-photon ATI cross section calculated using Coulomb waves is shown in Fig. 1. (Coulomb waves are the continuum eigenfunctions of an electron in the presence of a proton.) The rapid decrease in the $\hat{\mathbf{A}} \cdot \hat{\mathbf{p}}$-induced cross section with increasing photon energy may be qualitatively understood as follows. Two-photon ATI via $\hat{\mathbf{A}} \cdot \hat{\mathbf{p}}$ is a sequential two-step process. In the first step, the bound electron absorbs a photon and is excited to a continuum eigenstate; in the second step, the continuum electron absorbs a second photon to reach the final continuum state. At sufficiently high photon energy, the photoelectron in the intermediate and final states has a kinetic energy that is much higher than the $1 s$ binding energy. The Coulomb interaction between the photoelectron and the proton may then be neglected. This allows one to approximate $\Psi_{\tilde{\epsilon} p}$ and $\Psi_{f}$ by plane waves. (Indeed, it is known that the $1 s$ one-photon ionization cross sections calculated using plane waves and Coulomb waves, respectively, converge at high energies.) If we use plane waves for both intermediate and final states, then the $\hat{\mathbf{A}} \cdot \hat{\mathbf{p}}$-induced twophoton ATI cross section vanishes because a free electron cannot absorb a photon. Therefore, it is understandable that $\sigma_{\hat{\mathbf{A}} \cdot \hat{\mathbf{p}}}^{(2)}$ calculated using Coulomb waves vanishes rapidly as the photon energy increases.

Let us now turn to the $\hat{\mathbf{A}}^{2}$ contribution to the two-photon ATI cross section. After dropping the $\hat{\mathbf{A}} \cdot \hat{\mathbf{p}}$ term in Eq. (10), we obtain the $\hat{\mathbf{A}}^{2}$-induced two-photon ATI cross section

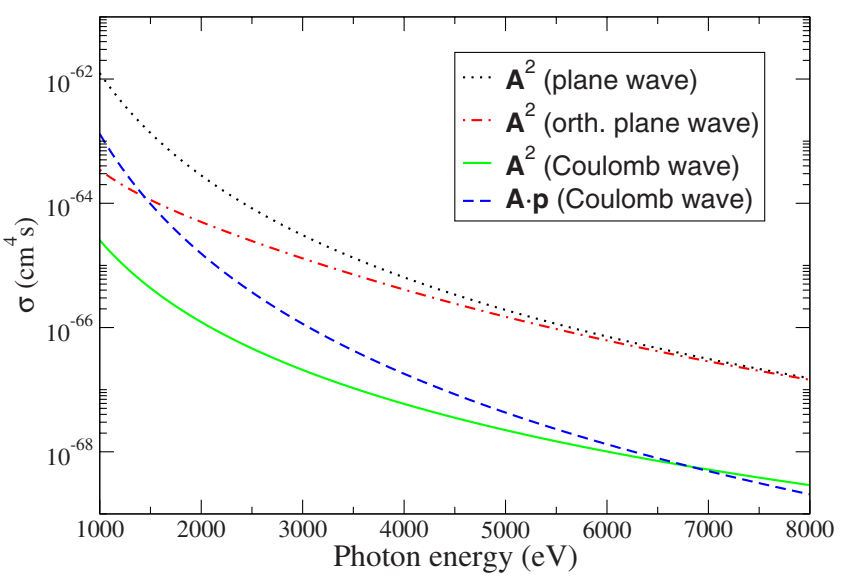

FIG. 2. (Color online) $\hat{\mathbf{A}}^{2}$-induced two-photon ATI cross section using three different final-state models. (i) Plane waves (dotted); (ii) orthogonalized plane waves (dot-dashed); and (iii) Coulomb waves (solid). Also shown is $\sigma_{\hat{\mathbf{A}} \cdot \hat{\mathbf{p}}}^{(2)}($ dashed).

$$
\sigma_{\hat{\mathbf{A}}^{2}}^{(2)}=\frac{2 \pi^{3} \alpha^{2}}{\omega^{2}} \sum_{l_{f}}\left(2 l_{f}+1\right)\left|R_{l_{f}}\right|_{\varepsilon_{f}=\varepsilon_{1 s}+2 \omega}^{2} .
$$

Here,

$$
R_{l_{f}}=\int u_{\varepsilon_{f} l_{f}}(r) j_{l_{f}}(2 k r) u_{1 s}(r) d r
$$

where $u_{1 s}(r)$ and $u_{\varepsilon_{f}, l_{f}}(r)$ are radial-wave functions for the initial and final states, respectively; $j_{l_{f}}(2 k r)$ is a spherical Bessel function. Note that there are no intermediate states involved in Eq. (13), so that there are no free-free transitions that might suppress the two-photon ATI cross section. However, the two-photon ATI via $\hat{\mathbf{A}}^{2}$ is a pure nondipole effect. In the electric dipole approximation, $k=0$. Thus, $j_{l_{f}}(2 k r) \rightarrow \delta_{l_{f}, 0}$. It follows that as a consequence of the orthogonality of $u_{1 s}$ and $u_{\varepsilon_{f}, 0}, R_{l_{f}}=0$ and, therefore, $\sigma_{\hat{\mathbf{A}}^{2}}^{(2)}=0$.

Based on our discussion above, it appears natural to adopt the plane-wave model for the final state in order to evaluate Eq. (13). The result is plotted in Fig. 1. Within the planewave model for $\sigma_{\hat{\mathbf{A}}^{2}}^{(2)}$, the $\hat{\mathbf{A}}^{2}$-induced two-photon ATI cross section is seen to dominate the numerically exact $\sigma_{\hat{\mathbf{A}} \cdot \hat{\mathbf{p}}}^{(2)}$, starting at photon energies as low as $50 \mathrm{eV}$. It is thus tempting to conclude that the two-photon ATI of hydrogen in the extreme ultraviolet is largely a nondipole effect. However, this is an artifact. The apparent importance of $\hat{\mathbf{A}}^{2}$ at relatively low photon energies is a consequence of the fact that a plane wave is not an eigenstate of the atomic Hamiltonian and is, therefore, not orthogonal to the $1 s$ state [37]. As explained earlier, the orthogonality between exact eigenstates is the reason why $\sigma_{\hat{\mathbf{A}}^{2}}^{(2)}$ vanishes at long wavelengths.

It is therefore necessary to orthogonalize the plane waves with respect to the $1 s$ state. This procedure ensures that $\sigma_{\hat{\mathbf{A}}^{2}}^{(2)}=0$ in the electric dipole approximation. In Fig. 2, we plot $\sigma_{\hat{\mathbf{A}}^{2}}^{(2)}$ calculated using orthogonalized plane waves. The orthogonalized-plane-wave cross section is smaller than the 
corresponding plane-wave cross section because orthogonalization removes artificial transitions that are, otherwise, allowed in the plane-wave model. As the photon energy increases, the orthogonalized-plane-wave model converges to the plane-wave model as expected. The consequence of orthogonalization can be seen at the crossing point, where the $\hat{\mathbf{A}}^{2}$ contribution starts dominating the $\hat{\mathbf{A}} \cdot \hat{\mathbf{p}}$ contribution. Orthogonalization pushes the crossing point from $50 \mathrm{eV}$ in the plane-wave model to a much higher energy of about 1500 $\mathrm{eV}$.

The orthogonalized-plane-wave model overestimates $\sigma_{\hat{\mathbf{A}}^{2}}^{(2)}$ by orders of magnitude, even at photon energies as high as 8 $\mathrm{keV}$. This is illustrated in Fig. 2, where we show $\sigma_{\hat{\mathbf{A}}^{2}}^{(2)}$ calculated using Coulomb waves. As may be seen in Fig. 2, between 1 and $8 \mathrm{keV}$, the numerically exact $\sigma_{\hat{\mathbf{A}}^{2}}^{(2)}$ is much smaller than the corresponding cross sections calculated using the two plane-wave models. At $8 \mathrm{keV}$, the cross sections differ by a factor of 50 . The Coulomb correction to the final states pushes the crossing point between $\sigma_{\hat{\mathbf{A}}^{2}}^{(2)}$ and $\sigma_{\hat{\mathbf{A}} \cdot \hat{\mathbf{p}}}^{(2)}$ further up in energy to $6815 \mathrm{eV}$.

The most dramatic effect of the dominance of $\hat{\mathbf{A}}^{2}$ at high photon energies is found in the photoelectron angular distribution. In the one-photon case, the nondipole corrections are relatively small, even at a photon energy of $8 \mathrm{keV}$. Thus, the photoelectron angular distribution is basically a dipole pattern directed along the polarization vector. Within the electric dipole approximation, the only allowed two-photon transitions induced by $\hat{\mathbf{A}} \cdot \hat{\mathbf{p}}$ are $1 s \rightarrow \varepsilon s$ and $1 s \rightarrow \varepsilon d$. In contrast, in our calculation using Coulomb waves, we find that the main contribution to $\sigma_{\hat{\mathbf{A}}^{2}}^{(2)}$ comes from $1 s \rightarrow \varepsilon p$. Two-photon absorption via $\hat{\mathbf{A}}^{2}$ leads to a dipolar angular distribution directed along the propagation vector $\mathbf{k}$. This is easily understood since $\mathbf{k}$ may be used to define the quantization axis when evaluating the matrix element $\left\langle\Psi_{f}\left|e^{2 i \mathbf{k} \cdot \mathbf{r}}\right| \Psi_{i}\right\rangle$ in Eq. (10). As a consequence of the fact that the $\hat{\mathbf{A}} \cdot \hat{\mathbf{p}}$ and $\hat{\mathbf{A}}^{2}$ terms in Eq. (10) lead to different final states, interference between the two terms in the evaluation of Eq. (10) may be neglected. Hence, to a good approximation, $\sigma_{\text {total }}^{(2)}=\sigma_{\hat{\mathbf{A}} \cdot \hat{\mathbf{p}}}^{(2)}+\sigma_{\hat{\mathbf{A}}^{2}}^{(2)}$.

\section{KINETIC MODEL}

At low intensities, ionization of light atoms, such as hydrogen or helium, by hard $\mathrm{x}$ rays is dominated by Compton scattering $[38,39]$, which is known to be a pure $\hat{\mathbf{A}}^{2}$ effect $[31,40]$. In order to assess the relative importance of $\mathrm{x}$-ray two-photon ATI in the high-intensity regime, we use the following rate equations:

$$
\begin{gathered}
\dot{p}^{(0)}(t)=-\left[\dot{p}^{(1)}(t)+\dot{p}^{(2)}(t)+\dot{p}^{(c)}(t)\right], \\
\dot{p}^{(1)}(t)=\sigma_{\text {total }}^{(1)} J(t) p^{(0)}(t), \\
\dot{p}^{(2)}(t)=\sigma_{\text {total }}^{(2)} J(t)^{2} p^{(0)}(t),
\end{gathered}
$$

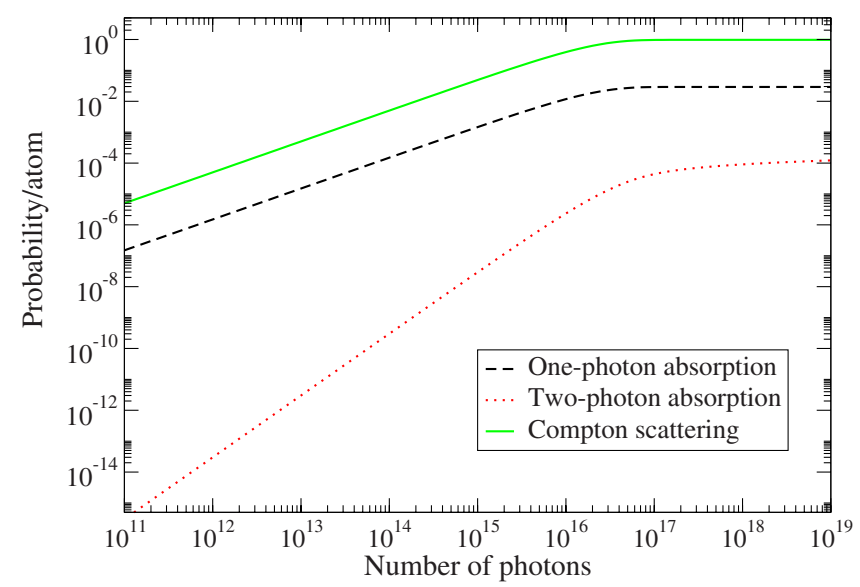

FIG. 3. (Color online) Probabilities, as a function of the number of photons per x-ray pulse, for ionization via (i) Compton scattering (solid), (ii) one-photon absorption (dashed), and (iii) two-photon absorption (dot-dashed). The photon energy is $6815 \mathrm{eV}$.

$$
\dot{p}^{(c)}(t)=\sigma_{\text {total }}^{(c)} J(t) p^{(0)}(t),
$$

to calculate, at a photon energy of $6815 \mathrm{eV}$, the probabilities per atom for one-photon absorption $\left[p^{(1)}\right]$, two-photon absorption $\left[p^{(2)}\right]$, and Compton scattering $\left[p^{(c)}\right] ; p^{(0)}$ is the probability to find the atom in its ground state. These rate equations are numerically solved using the fourth-order Runge-Kutta algorithm. The Compton scattering cross section $\sigma^{(c)}$ for hydrogen is taken from the NIST XCOM database [41]. We assume a Gaussian X-ray pulse with a duration of 2 fs full width at half maximum [42] and a focal width of $1 \mu \mathrm{m}$ full width at half maximum. The probabilities for the three different ionization channels, as a function of the number of photons in the X-ray pulse, are plotted in Fig. 3.

At $10^{12}$ photons, the Compton scattering probability is $5.0 \times 10^{-5}$, whereas the one- and two-photon absorption probabilities are $1.5 \times 10^{-6}$ and $3.0 \times 10^{-14}$, respectively. With increasing photon number, the relative importance of two-photon ATI increases. Saturation of ionization occurs near a photon number of $10^{17}$, where the two-photon ATI probability is $4.5 \times 10^{-5}$. Nevertheless, as may be seen in Fig. 3, ionization through Compton scattering is the most important process throughout.

\section{FIELD COHERENCE}

As in the case of optical light $[43,44]$, nonlinear processes in the $\mathrm{x}$-ray regime depend on the generalized coherence properties of the radiation source $[45,46]$. The LCLS and other forthcoming X-ray free-electron-laser sources are based on the self-amplified spontaneous emission (SASE) scheme $[47,48]$, thus, providing nonreproducible quasichaotic pulses of highly fluctuating intensity in both the temporal and spectral domains. Each SASE pulse consists of a train of phaseuncorrelated intensity spikes of random pulse height and duration $[49,50]$. These intensity spikes will enhance multiphoton effects, as compared to coherent radiation with the same average intensity. The importance of this effect can easily be assessed by modeling the SASE radiation by cha- 
otic radiation. This approach is valid for SASE radiation in the exponential gain regime [51]. The impact of the properties of SASE radiation on X-ray two-photon absorption mediated by second-order $\hat{\mathbf{A}} \cdot \hat{\mathbf{p}}$ is discussed in Ref. [11].

Applying a density-matrix approach for the radiation field, as described in Ref. [11], and assuming that the ground-state population remains essentially unmodified by the $\mathrm{x}$ rays, it can be shown that the probability of an $\hat{\mathbf{A}}^{2}$-induced two-photon absorption event is given by

$$
p_{\hat{\mathbf{A}}^{2}}^{(2)}=\sigma_{\hat{\mathbf{A}}^{2}}^{(2)} \int_{-\infty}^{\infty}\left\langle J(t)^{2}\right\rangle d t,
$$

where $\left\langle J(t)^{2}\right\rangle$ denotes the ensemble-averaged second moment of the x-ray flux $J(t)$. For SASE light, modeled by chaotic radiation,

$$
\left\langle J(t)^{2}\right\rangle=2\langle J(t)\rangle^{2}
$$

as a consequence of the Siegert relations, which relate higher-order correlation functions of the field to the firstorder correlation function $[45,52]$. Hence, the $\hat{\mathbf{A}}^{2}$-mediated two-photon ATI process, when driven by a SASE freeelectron laser, will be enhanced by a factor of 2 compared to using a smooth ensemble-averaged pulse profile. This enhancement factor is too small to change the conclusions drawn in the previous section.

\section{SUMMARY}

In conclusion, we have investigated the nature of $\mathrm{x}$-ray two-photon ATI of atomic hydrogen. We have found that at photon energies higher than $6.8 \mathrm{keV}$, two-photon ATI is mediated primarily by $\hat{\mathbf{A}}^{2}$. This renders two-photon ATI at high photon energies essentially a pure nondipole effect. Also, above $6.8 \mathrm{keV}$, two-photon ATI becomes fundamentally nonsequential, as there are no intermediate states involved in the $\hat{\mathbf{A}}^{2}$-mediated two-photon absorption process. This behavior at high photon energies is totally different from two-photon ATI at longer wavelengths, which is a two-step process that may be described within the dipole approximation. The failure of the plane-wave model has a significant impact on the crossing point between the $\hat{\mathbf{A}}^{2}$ and $\hat{\mathbf{A}} \cdot \hat{\mathbf{p}}$ contributions to the two-photon ATI cross section. The Coulomb corrections to the wave function of the ejected electron cause $\sigma_{\hat{\mathbf{A}}^{2}}^{(2)}$ to be greater than $\sigma_{\hat{\mathbf{A}} \cdot \hat{\mathbf{p}}}^{(2)}$ only at photon energies that are so high that ionization of hydrogen is dominated by Compton scattering.

\section{ACKNOWLEDGMENTS}

H.V. and R.S. were supported by the Office of Basic Energy Sciences, Office of Science, U.S. Department of Energy, under Contract No. DE-AC02-06CH11357. M.F.C. acknowledges the Visitor Program of the Max Planck Institute for the Physics of Complex Systems. Part of this work was performed under the auspices of the U.S. Department of Energy by Lawrence Livermore National Laboratory under Contract No. DE-AC52-07NA27344.
[1] J. Feldhaus, J. Arthur, and J. B. Hastings, J. Phys. B 38, S799 (2005).

[2] J. Arthur et al., Linac Coherent Light Source (LCLS) Conceptual Design Report No. SLAC-R-593, 2002 (unpublished); http://www-ssrl.slac.stanford.edu/lcls/cdr/

[3] T. Tanaka and T. Shintake, SCSS X-FEL Conceptual Design Report (Riken, Japan, May 2005); http://wwwxfel.spring8.or.jp/

[4] M. Altarelli et al., The European X-ray Free Electron Laser Technical Design Report No. DESY 2006-097, 2006 (unpublished); http://xfel.desy.de/tdr/index_eng.html

[5] M. A. Kornberg, A. L. Godunov, S. Itza-Ortiz, D. L. Ederer, J. H. McGuire, and L. Young, J. Synchrotron Radiat. 9, 298 (2002).

[6] H. N. Chapman, A. Barty, M. J. Bogan, S. Boutet, M. Frank, S. P. Hau-Riege, S. Marchesini, B. W. Woods, S. Bajt, W. H. Benner, R. A. London, E. Plönjes, M. Kuhlmann, R. Treusch, S. Düsterer, T. Tschentscher, J. R. Schneider, E. Spiller, T. Möller, C. Bostedt, M. Hoener, D. A. Shapiro, K. O. Hodgson, D. van der Spoel, F. Burmeister, M. Bergh, C. Caleman, G. Huldt, M. M. Seibert, F. R. N. C. Maia, R. W. Lee, A. Szöke, N. Timneanu, and J. Hajdu, Nat. Phys. 2, 839 (2006).

[7] U. Saalmann and J.-M. Rost, Phys. Rev. Lett. 89, 143401 (2002).

[8] R. Neutze, R. Wouts, D. Spoel, E. Weckert, and J. Hajdu,
Nature (London) 406, 752 (2000)

[9] G. Faigel, Z. Jurek, G. Oszlanyi, and M. Tegze, J. Alloys Compd. 401, 86 (2005).

[10] S. P. Hau-Riege, R. A. London, H. N. Chapman, A. Szoke, and N. Timneanu, Phys. Rev. Lett. 98, 198302 (2007).

[11] N. Rohringer and R. Santra, Phys. Rev. A 76, 033416 (2007).

[12] M. G. Makris, P. Lambropoulos, and A. Mihelič, Phys. Rev. Lett. 102, 033002 (2009).

[13] R. Santra, N. V. Kryzhevoi, and L. S. Cederbaum, Phys. Rev. Lett. 103, 013002 (2009).

[14] P. Agostini, F. Fabre, G. Mainfray, G. Petite, and N. K. Rahman, Phys. Rev. Lett. 42, 1127 (1979).

[15] R. R. Freeman, P. H. Bucksbaum, H. Milchberg, S. Darack, D. Schumacher, and M. E. Geusic, Phys. Rev. Lett. 59, 1092 (1987).

[16] R. M. Potvliege and S. Vučić, J. Phys. B 42, 055603 (2009).

[17] B. Milošević, G. G. Paulus, D. Bauer, and W. Becker, J. Phys. B 39, R203 (2006).

[18] L. F. DiMauro and P. Agostini, Adv. At., Mol., Opt. Phys. 35, 79 (1995).

[19] N. Miyamoto, M. Kamei, D. Yoshitomi, T. Kanai, T. Sekikawa, T. Nakajima, and S. Watanabe, Phys. Rev. Lett. 93, 083903 (2004).

[20] M. Meyer, D. Cubaynes, D. Glijer, J. Dardis, P. Hayden, P. Hough, V. Richardson, E. T. Kennedy, J. T. Costello, P. Radc- 
liffe, S. Düsterer, A. Azima, W. B. Li, H. Redlin, J. Feldhaus, R. Taïeb, A. Maquet, A. N. Grum-Grzhimailo, E. V. Gryzlova, and S. I. Strakhova, Phys. Rev. Lett. 101, 193002 (2008).

[21] A. R. B. de Castro, T. Laarmann, J. Schulz, H. Wabnitz, and T. Möller, Phys. Rev. A 72, 023410 (2005).

[22] D. P. Craig and T. Thirunamachandran, Molecular Quantum Electrodynamics (Dover, Mineola, New York, 1998).

[23] P. Koval, S. Fritzsche, and A. Surzhykov, J. Phys. B 37, 375 (2004).

[24] G. Çelik, E. Çelik, and H. Ş. Kiliç, Eur. Phys. J. D 50, 237 (2008).

[25] I. A. Ivanov and A. S. Kheifets, Radiat. Phys. Chem. 75, 2102 (2006).

[26] R. Radhakrishnan and R. B. Thayyullathil, Phys. Rev. A 69, 033407 (2004).

[27] P. Antoine, N.-E. Essarroukh, J. Jureta, X. Urbain, and F. Brouillard, J. Phys. B 29, 5367 (1996).

[28] H. Rottke, B. Wolff, M. Brickwedde, D. Feldmann, and K. H. Welge, Phys. Rev. Lett. 64, 404 (1990).

[29] R. S. D. Sihombing, M. Katsuragawa, G. Z. Zhang, and K. Hakuta, Phys. Rev. A 54, 1551 (1996).

[30] S. Raimes, Many-Electron Theory (North-Holland, Amsterdam, 1972).

[31] R. Santra, J. Phys. B 42, 023001 (2009); 42, 169801 (2009).

[32] H. Friedrich, Theoretical Atomic Physics (Springer-Verlag, Berlin, 2006).

[33] B. Gao and A. F. Starace, Comput. Phys. 1, 70 (1987).

[34] M. Førre, J. P. Hansen, L. Kocbach, S. Selstø, and L. B. Madsen, Phys. Rev. Lett. 97, 043601 (2006).

[35] S. Selst $\varnothing$ and M. Førre, Phys. Rev. A 76, 023427 (2007).

[36] M. Førre, S. Selstø, J. P. Hansen, and L. B. Madsen, Phys. Rev. Lett. 95, 043601 (2005).
[37] A. Maquet, B. Piraux, A. Scrinzi, and R. Taïeb, Phys. Rev. A 74, 027401 (2006).

[38] H. A. Bethe and E. E. Salpeter, Quantum Mechanics of Oneand Two-Electron Atoms (Dover, Mineola, New York, 2008).

[39] J. A. R. Samson, C. H. Greene, and R. J. Bartlett, Phys. Rev. Lett. 71, 201 (1993).

[40] P. Eisenberger and P. M. Platzman, Phys. Rev. A 2, 415 (1970).

[41] http://physics.nist.gov/PhysRefData/Xcom/Text/XCOM.html

[42] Y. Ding, A. Brachmann, F.-J. Decker, D. Dowell, P. Emma, J. Frisch, S. Gilevich, G. Hays, Ph. Hering, Z. Huang, R. Iverson, H. Loos, A. Miahnahri, H.-D. Nuhn, D. Ratner, J. Turner, J. Welch, W. White, and J. Wu, Phys. Rev. Lett. 102, 254801 (2009).

[43] P. Lambropoulos, C. Kikuchi, and R. K. Osborn, Phys. Rev. 144, 1081 (1966).

[44] B. R. Mollow, Phys. Rev. 175, 1555 (1968).

[45] R. J. Glauber, Phys. Rev. 130, 2529 (1963).

[46] R. J. Glauber, Phys. Rev. 131, 2766 (1963).

[47] A. M. Kondratenko and E. L. Saldin, Sov. Phys. Dokl. 24, 986 (1979).

[48] R. Bonifacio, C. Pellegrini, and L. M. Narducci, Opt. Commun. 50, 373 (1984).

[49] E. L. Saldin, E. A. Schneidmiller, and M. V. Yurkov, Opt. Commun. 148, 383 (1998).

[50] E. L. Saldin, E. A. Schneidmiller, and M. V. Yurkov, The Physics of Free Electron Lasers (Springer-Verlag, Berlin, 2000).

[51] S. Krinsky and R. L. Gluckstern, Phys. Rev. ST Accel. Beams 6, 050701 (2003).

[52] B. Saleh, Photoelectron Statistics (Springer-Verlag, Berlin, 1978). 